\title{
Alternative method for deriving nonlocal multipartite product states
}

\author{
Mao-Sheng Li \\ Department of Mathematical Sciences, Tsinghua University, Beijing100084, China \\ Yan-Ling Wang* \\ School of Computer Science and Network Security, \\ Dongguan University of Technology, Dongguan 523808, China
}

\begin{abstract}
We can only perform a finite rounds of measurements in protocols with local operations and classical communication (LOCC). In this paper, we propose a set of product states, which require infinite rounds of measurements in order to distinguish this given set of states perfectly by LOCC. Therefore, we can conclude that these sets of states are locally indistinguishable. More accurately, given any multipartite LOCC indistinguishable set where every local system cannot start with a nontrivial measurement, then after appending these states with arbitrarily choosing two nonorthogonal states, we obtain another LOCC indistinguishable set. It can be seen that some parties can perform some nontrivial measurements. Hence, these sets are quite different from those constructed before. This result broadens the knowledge of nonlocality without entanglement to a certain extent.
\end{abstract}

PACS numbers: 03.67.Hk,03.65.Ud

\section{INTRODUCTION}

In quantum information theory, quantum information is always hidden in the quantum states. In particular, classical information can also be encoded within a set of finitely chosen quantum states. The decoding process can be seen as a protocol that could distinguish the encoded quantum states. It is well known that a given set of quantum states can be perfectly identified if and only if the states of the given set are mutually orthogonal [1]. However, we often encounter compound quantum systems, and the physical conditions always restrict our ability such that we are only allowed to do local operations and classical communication (LOCC). In fact, there exist many global operators that cannot be implemented by LOCC. Hence, it is fundamentally of interest to study the following problem: given a set of mutually orthogonal states in compound quantum systems, can these given states be identified only using LOCC? If so, we call them locally distinguishable or distinguishable by LOCC. If not, we call them locally indistinguishable or indistinguishable by LOCC. These states are also known as a nonlocal set in the latter case. The local distinguishability has been practically applied in quantum cryptography primitives such as secret sharing and data hiding $[2,3]$.

Maximally entangled states and product states have been mostly studied. References [4-15] provide an incomplete list of the results about the local distinguishability of maximally entangled states. At the same time, many people have considered the local distinguishability of product states [16-33].

The case of product states was first considered by Bennett. et al., who presented nine LOCC indistinguishable product states in $\mathbb{C}^{3} \otimes \mathbb{C}^{3}[16]$. Since then, the local indistinguishability of orthogonal product states in bipartite

\footnotetext{
*wangylmath@yahoo.com
}

systems has attracted much attention [21-24, 26, 27, 29]. More recently, the study of multipartite quantum systems has attracted increased attention [25, 28, 31, 33]. More precisely, $\mathrm{Xu}$ et al. presented a small set with $2 n$ locally indistinguishable multipartite orthogonal product states in $\mathbb{C}^{d_{1}} \otimes \mathbb{C}^{d_{2}} \otimes \cdots \otimes \mathbb{C}^{d_{n}}$ for $n \geq 3$. Subsequently, Wang et al. gave an explicit construction of locally indistinguishable multipartite orthogonal product states for multipartite quantum systems using a set of locally indistinguishable bipartite orthogonal product states. Zhang et al. provided general methods to construct locally indistinguishable orthogonal product states for multipartite quantum systems using a set of locally indistinguishable bipartite orthogonal product states with the following additional condition: two partites cannot start a nontrivial measurement. More recently, S. Halder presented several sets that no states can be eliminated from the basis by performing orthogonality preserving measurements [33]. Another direction of related research is to study entanglement as a resource to distinguish quantum states of locally indistinguishable states [34-36].

Note that for almost all these results, except the unextendible product basis(UPB) [19, 20, 37-39], their construction is based on useful techniques developed by Walgate and Hardy [5]. Hence, it is fundamentally of interest to find some other methods that would lead to more abundant constructions of nonlocal product states.

The rest of the article is organized as follows. In Sec. II, we give some necessary definitions and some important concepts. In Sec. III, we present our main results. We start with a simple example to show the main idea of our method. Then we show a much more general statement. For some given set of mutually orthogonal product states which is LOCC indistinguishable because all the local systems can not start with a nontrivial measurement, we can extend it by another partite whose local state is selected from a set with two nonorthogonal states. Although with one more partite joined in, the set is still 
LOCC indistinguishable. After that, we generalize this with more parties joined in and show the target set is still LOCC indistinguishable. At last, we give an example to show that our theorems can not be "simply generalized" to a set of three or more nonorthogonal states. Finally, we draw a conclusion and present some interesting problems in section IV.

\section{PRELIMINARIES}

To perfectly distinguish a set of states, these states must be orthogonal to each other. Based on this fact, we know that only orthogonality-preserving measurements are allowed to perfectly distinguish a set of orthogonal states by LOCC. Most locally indistinguishable product states are based on the principle that there must exist some partite who can be the first to perform a nontrivial measurement. Here, we first give the definition of trivial measurement.

Definition 1. A measurement $\left\{M_{i}\right\}_{i=1}^{n}$ corresponding to a system of level $d$ is called a trivial measurement if

$$
\sum_{i=1}^{n} M_{i}^{\dagger} M_{i}=I_{d} \text { and } \quad M_{i}^{\dagger} M_{i} \propto I_{d}
$$

for any $1 \leq i \leq n$.

In order to perfectly distinguish a set of orthogonal states in multipartite quantum systems, any measurement should preserve the orthogonality relations of given states. The following six states in $\mathbb{C}^{2} \otimes \mathbb{C}^{2} \otimes \mathbb{C}^{2}$ can be proved to be LOCC indistinguishable, since no partite could start with a nontrivial measurement in order to preserve the orthogonality relations of these given states (See Refs. [25, 29]).

$$
\begin{aligned}
\left|\psi_{1}\right\rangle & =|0\rangle|1\rangle|0+1\rangle, \\
\left|\psi_{2}\right\rangle & =|0\rangle|1\rangle|0-1\rangle, \\
\left|\psi_{3}\right\rangle & =|0+1\rangle|0\rangle|1\rangle, \\
\left|\psi_{4}\right\rangle & =|0-1\rangle|0\rangle|1\rangle, \\
\left|\psi_{5}\right\rangle & =|1\rangle|0+1\rangle|0\rangle, \\
\left|\psi_{6}\right\rangle & =|1\rangle|0-1\rangle|0\rangle .
\end{aligned}
$$

Definition 2. Let $\mathcal{H}$ denote an $m$ partite quantum system $\otimes_{i=1}^{m} \mathcal{H}_{i}$. Suppose $\mathcal{S}$ is a set of mutually orthogonal product states in $\mathcal{H}$. We say $\mathcal{S}$ is completable in $\mathcal{H}$ if its complementary subspace $\mathcal{S}^{\perp}$ is spanned by a set of orthogonal product states. Equivalently, there exists a product basis $\mathcal{B}$ of $\mathcal{H}$ such that $\mathcal{S} \subseteq \mathcal{B}$.

\section{METHOD FOR DERIVING NONLOCAL MULTIPARTITE PRODUCT STATES}

It is well known that a set $\mathcal{S}$ of quantum states can be distinguished by global operations if and only if the states in $\mathcal{S}$ are pairwise orthogonal to each other. The following lemma illustrates the most important reason why two nonorthogonal quantum states cannot be distinguished. This lemma will be used repeatedly in this article. So we also give a detail proof of it. But for the sake of readability of the article we leave it in APPENDIX A.

Lemma 1. Let $|\alpha\rangle,|\beta\rangle$ be two nonorthogonal states in $\mathbb{C}^{d}$. For any measurement $\mathcal{M}=\left\{M_{i}\right\}_{i=1}^{n}$, there exists some $i$ such that the following three terms are nonzero:

$$
\left\langle\alpha\left|M_{i}^{\dagger} M_{i}\right| \alpha\right\rangle,\left\langle\alpha\left|M_{i}^{\dagger} M_{i}\right| \beta\right\rangle,\left\langle\beta\left|M_{i}^{\dagger} M_{i}\right| \beta\right\rangle .
$$

That is, the post-measurement states of the outcome $i$ are still nonorthogonal states.

Now we begin to discuss the main issues we will consider. Notice that any five of the six states in equation (1) are LOCC distinguishable. Therefore, it is easy to see that the following six states

$$
\left|\psi_{1}\right\rangle\left|\alpha_{1}\right\rangle,\left|\psi_{2}\right\rangle\left|\alpha_{2}\right\rangle,\left|\psi_{3}\right\rangle\left|\alpha_{3}\right\rangle,\left|\psi_{4}\right\rangle\left|\alpha_{4}\right\rangle,\left|\psi_{5}\right\rangle\left|\alpha_{5}\right\rangle,\left|\psi_{6}\right\rangle\left|\alpha_{6}\right\rangle
$$

are LOCC distinguishable if two of the six states $\left|\alpha_{1}\right\rangle$, $\left|\alpha_{2}\right\rangle,\left|\alpha_{3}\right\rangle,\left|\alpha_{4}\right\rangle,\left|\alpha_{5}\right\rangle,\left|\alpha_{6}\right\rangle$ are orthogonal to each other. It is interesting to consider the following case: Suppose $\left|\alpha_{1}\right\rangle,\left|\alpha_{2}\right\rangle,\left|\alpha_{3}\right\rangle,\left|\alpha_{4}\right\rangle,\left|\alpha_{5}\right\rangle,\left|\alpha_{6}\right\rangle$ are not orthogonal to each other, then what result about the local distinguishability of states in (2) can we obtain? Here we start with a simple example.

Example 1. With the same notation in equation (1), and let $|\alpha\rangle,|\beta\rangle$ be any two nonorthogonal states in $\mathbb{C}^{2}$. Then the set of states

$$
\mathcal{S}:=\left\{\left|\psi_{1}\right\rangle|\alpha\rangle,\left|\psi_{2}\right\rangle|\alpha\rangle,\left|\psi_{3}\right\rangle|\alpha\rangle,\left|\psi_{4}\right\rangle|\beta\rangle,\left|\psi_{5}\right\rangle|\beta\rangle,\left|\psi_{6}\right\rangle|\beta\rangle\right\}
$$

in $\mathbb{C}^{2} \otimes \mathbb{C}^{2} \otimes \mathbb{C}^{2} \otimes \mathbb{C}^{2}$ is LOCC indistinguishable .

Proof: Denote

$$
\left|\alpha_{1}\right\rangle=\left|\alpha_{2}\right\rangle=\left|\alpha_{3}\right\rangle=|\alpha\rangle,\left|\alpha_{4}\right\rangle=\left|\alpha_{5}\right\rangle=\left|\alpha_{6}\right\rangle=|\beta\rangle .
$$

Since the last partite of states in $\mathcal{S}$ are not orthogonal to each other, we can show that all the first three parties could only start with a trivial measurement in order to preserve the orthogonality relations of states in $\mathcal{S}$. For instance, if the first partite goes first in the distinguishing protocol. Let $M^{\dagger} M$ be one of the POVM matrix performed by the first partite. The orthogonal relations

$$
\left\langle\alpha_{i}\left|\left\langle\psi_{i}\left|M^{\dagger} M \otimes I_{2} \otimes I_{2} \otimes I_{2}\right| \psi_{j}\right\rangle\right| \alpha_{j}\right\rangle=0, \text { for all } i \neq j
$$

are just equivalent with

$$
\left\langle\psi_{i}\left|M^{\dagger} M \otimes I_{2} \otimes I_{2}\right| \psi_{j}\right\rangle=0, \text { for all } i \neq j .
$$

However, using the latter relations, we can deduce that $M^{\dagger} M \propto I_{2}$, i.e., the first partite could only start with trivial measurement.

Hence, the fourth partite should be the first one to take a nontrivial measurement $\mathcal{M}=\left\{M_{i}\right\}_{i=1}^{n}$. By lemma 1, there exists at least one $i$ such that $\left|\alpha^{\prime}\right\rangle=M_{i}|\alpha\rangle,\left|\beta^{\prime}\right\rangle=$ 
$M_{i}|\beta\rangle$ are not orthogonal to each other. Normalizing the two states $\left|\alpha^{\prime}\right\rangle,\left|\beta^{\prime}\right\rangle$ to be $\left|\widehat{\alpha}^{\prime}\right\rangle,\left|\widehat{\beta}^{\prime}\right\rangle$, we redenote them to be $\left|\alpha^{\prime}\right\rangle,\left|\beta^{\prime}\right\rangle$. After the first round of measurement with the outcome " $i$ ", the post-measurement states are just

$$
\mathcal{S}^{\prime}=\left\{\left|\psi_{1}\right\rangle\left|\alpha^{\prime}\right\rangle,\left|\psi_{2}\right\rangle\left|\alpha^{\prime}\right\rangle,\left|\psi_{3}\right\rangle\left|\alpha^{\prime}\right\rangle,\left|\psi_{4}\right\rangle\left|\beta^{\prime}\right\rangle,\left|\psi_{5}\right\rangle\left|\beta^{\prime}\right\rangle,\left|\psi_{6}\right\rangle\left|\beta^{\prime}\right\rangle\right\} .
$$

In order to perfectly distinguish the states $\mathcal{S}$, we have to perfectly distinguish the states $\mathcal{S}^{\prime}$. When compared the set $\mathcal{S}$ with $\mathcal{S}^{\prime}$, we realize that they share the same property : the first three local systems cannot start with a nontrivial measurement, and the local states of the fourth system are just chosen from a set with two nonorthogonal states.

With a similar argument for the set $\mathcal{S}^{\prime}$, in the second round of measurement, there exists an outcome " $j$ " of the fourth system measurement such that the postmeasurement states of the last partite are still nonorthogonal. This situation leads to another set

$\mathcal{S}^{\prime \prime}=\left\{\left|\psi_{1}\right\rangle\left|\alpha^{\prime \prime}\right\rangle,\left|\psi_{2}\right\rangle\left|\alpha^{\prime \prime}\right\rangle,\left|\psi_{3}\right\rangle\left|\alpha^{\prime \prime}\right\rangle,\left|\psi_{4}\right\rangle\left|\beta^{\prime \prime}\right\rangle,\left|\psi_{5}\right\rangle\left|\beta^{\prime \prime}\right\rangle,\left|\psi_{6}\right\rangle\left|\beta^{\prime \prime}\right\rangle\right\}$.

being distinguished. Noticing that the set $\mathcal{S}^{\prime \prime}$ shares the same property as the original set $\mathcal{S}$.

The significant point is that the above process cannot stop in any finite rounds of measurements. However, we are only allowed to perform a finite rounds of measurements. Therefore, we deduce that the given set $\mathcal{S}$ is indeed LOCC indistinguishable.

The above example shows the main idea about how to construct a nonlocal set of product states. In fact, we can consider a much more general setting.

Theorem 1. Let $\mathcal{S}_{o}=\left\{\left|\psi_{i}\right\rangle \mid 1 \leq i \leq k\right\}$ be a set of $m u$ tually orthogonal product states in $\mathbb{C}^{d_{1}} \otimes \mathbb{C}^{d_{2}} \otimes \cdots \otimes \mathbb{C}^{d_{m}}$. Suppose the set $\mathcal{S}_{o}$ is LOCC indistinguishable because all the local systems can not start with a nontrivial measurement to preserve the orthogonality of these given states. Let $|\alpha\rangle,|\beta\rangle$ be two nonorthogonal states in $\mathbb{C}^{d_{m+1}}$. Then the set of states $\mathcal{S}:=\left\{\left|\psi_{1}\right\rangle\left|\alpha_{1}\right\rangle, \ldots,\left|\psi_{k}\right\rangle\left|\alpha_{k}\right\rangle\right\}$ is also LOCC indistinguishable as $m+1$ parties with $\left|\alpha_{1}\right\rangle, \cdots,\left|\alpha_{k}\right\rangle \in\{|\alpha\rangle,|\beta\rangle\}$.

Proof: By assumption, each of the first $m$ parties cannot be the first one to take a nontrivial measurement. Hence, the $(m+1)$-th system should be the first one to perform a nontrivial measurement $\mathcal{M}=\left\{M_{i}\right\}_{i=1}^{n}$. By lemma 1, there exists at least one $i$ such that $\left|\alpha^{\prime}\right\rangle=M_{i}|\alpha\rangle,\left|\beta^{\prime}\right\rangle=$ $M_{i}|\beta\rangle$ are not orthogonal to each other. Normalizing the two states $\left|\alpha^{\prime}\right\rangle,\left|\beta^{\prime}\right\rangle$ to be $\left|\widehat{\alpha}^{\prime}\right\rangle,\left|\widehat{\beta}^{\prime}\right\rangle$, we redenote them to be $\left|\alpha^{\prime}\right\rangle,\left|\beta^{\prime}\right\rangle$.

After the first round of measurement with the outcome " $i$ ", the post-measurement states are just

$$
\mathcal{S}^{\prime}=\left\{\left|\psi_{1}\right\rangle\left|\alpha_{1}^{\prime}\right\rangle, \ldots,\left|\psi_{k}\right\rangle\left|\alpha_{k}^{\prime}\right\rangle\right\}
$$

with $\left|\alpha_{1}^{\prime}\right\rangle, \ldots,\left|\alpha_{k}^{\prime}\right\rangle \in\left\{\left|\alpha^{\prime}\right\rangle,\left|\beta^{\prime}\right\rangle\right\}$. In order to perfectly distinguish the states $\mathcal{S}$, we have to perfectly distinguish the states $\mathcal{S}^{\prime}$. It is obvious that $\mathcal{S}^{\prime}$ shares the same properties with $\mathcal{S}$ including the following: the first $m$ local parts cannot start with a nontrivial measurement, and the local states of the $(m+1)$-th system are just chosen from a set with two nonorthogonal states. With a similar argument for the set $\mathcal{S}^{\prime}$, there exists an outcome " $j$ " of $(m+1)$-th system measurement such that the post-measurement states of the last partite are still nonorthogonal. This situation leads to another set $\mathcal{S}^{\prime \prime}$ being distinguished, which shares the same property as the original set $\mathcal{S}$. And this process cannot stop in any finite rounds of measurements. However, we are only allowed to perform finite measurement rounds. To obtain a concise observation of the proof, we show a more intuitive figure (see Fig. 1). Hence, we deduce that the given set $\mathcal{S}$ is indeed LOCC indistinguishable.

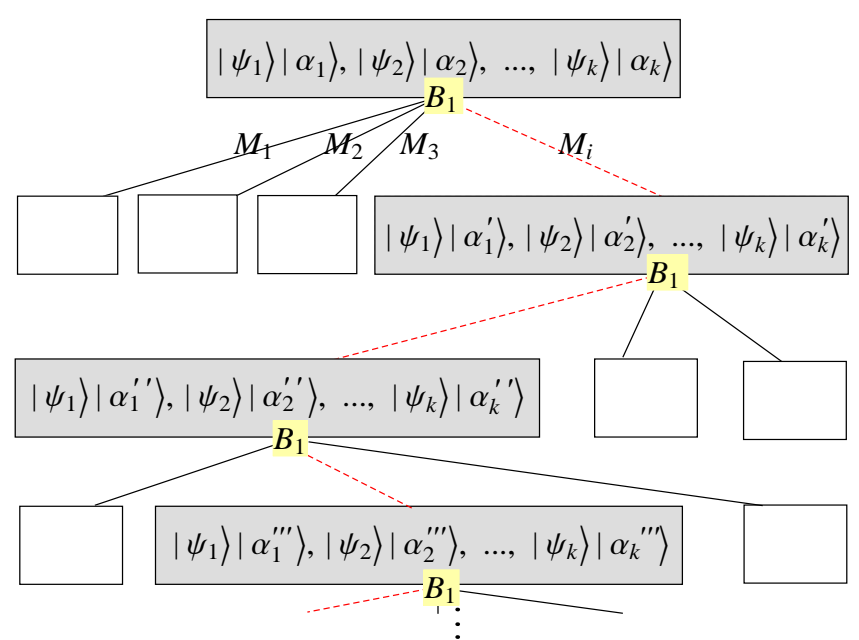

Fig. 1: This is a sketch map to show the distinguishing protocol of theorem 1. Here we use $A_{1}, A_{2}, \ldots, A_{m}$ to denote the first $m$ parties and $B_{1}$ to denote the last partite. There exists an infinite sequence of outcomes (indicated by the red dotted line) performed by the last partite. Moreover, the post-measurement states along these outcomes are pictured by the grey rectangle.

Denote $|S|$ to be the cardinality of a set $S$. The two sets $\mathcal{S}_{o}, \mathcal{S}$ in theorem 1 satisfy $\left|\mathcal{S}_{o}\right|=|\mathcal{S}|$. It is interesting to construct LOCC indistinguishable set with vary number of states. Based on the current scheme, we give some results related to this problem.

We maximally extent $\left\{\left|\psi_{1}\right\rangle, \ldots,\left|\psi_{k}\right\rangle\right\}$ by orthogonal product states in $\mathbb{C}^{d_{1}} \otimes \mathbb{C}^{d_{2}} \otimes \cdots \otimes \mathbb{C}^{d_{m}}$ and denote such a target set to be $\mathcal{S}_{o}^{\prime}=\left\{\left|\psi_{1}\right\rangle, \ldots,\left|\psi_{k}\right\rangle,\left|\psi_{k+1}\right\rangle \ldots,\left|\psi_{N}\right\rangle\right\}$. That is, the states in $\mathcal{S}_{o}^{\prime}$ are mutually orthogonal product states and there is no nonzero product state which is orthogonal to all the states of $\mathcal{S}_{o}^{\prime}$. If $N=k$, then $\mathcal{S}_{o}$ itself is an UPB (whose definition can be found in Ref. [19]). If $N=\prod_{i=1}^{m} d_{i}$, then $\mathcal{S}_{o}$ is completable. Generally speaking, $N$ lies between $k$ and $\prod_{i=1}^{m} d_{i}$. Let $\left\{\left|e_{1}\right\rangle,\left|e_{2}\right\rangle, \cdots,\left|e_{d_{m+1}}\right\rangle\right\}$ be an orthonormal basis of $\mathbb{C}^{d_{m+1}}$ with

$$
\left|e_{1}\right\rangle=|\alpha\rangle, \text { and } \operatorname{span}_{\mathbb{C}}\left\{\left|e_{1}\right\rangle,\left|e_{2}\right\rangle\right\}=\operatorname{span}_{\mathbb{C}}\{|\alpha\rangle,|\beta\rangle\} .
$$

Denote $\left|\alpha^{\perp}\right\rangle,\left|\beta^{\perp}\right\rangle$ to be the unique states (up to a phase) in $\operatorname{span}_{\mathbb{C}}\{|\alpha\rangle,|\beta\rangle\}$ which is orthogonal to $|\alpha\rangle,|\beta\rangle$, respec- 
tively. Then the following set $\mathcal{S}_{\text {ext }}$ of product states in $\mathbb{C}^{d_{1}} \otimes \mathbb{C}^{d_{2}} \otimes \cdots \otimes \mathbb{C}^{d_{m}} \otimes \mathbb{C}^{d_{m+1}}$

$$
\begin{aligned}
& \left|\psi_{1}\right\rangle\left|\alpha_{1}\right\rangle, \ldots,\left|\psi_{k}\right\rangle\left|\alpha_{k}\right\rangle,\left|\psi_{k+1}\right\rangle|\alpha\rangle, \ldots,\left|\psi_{N}\right\rangle|\alpha\rangle, \\
& \left|\psi_{1}\right\rangle\left|\alpha_{1}^{\perp}\right\rangle, \ldots,\left|\psi_{k}\right\rangle\left|\alpha_{k}^{\perp}\right\rangle,\left|\psi_{k+1}\right\rangle\left|\alpha^{\perp}\right\rangle, \ldots,\left|\psi_{N}\right\rangle\left|\alpha^{\perp}\right\rangle, \\
& \left|\psi_{1}\right\rangle\left|e_{3}\right\rangle, \ldots,\left|\psi_{k}\right\rangle\left|e_{3}\right\rangle,\left|\psi_{k+1}\right\rangle\left|e_{3}\right\rangle, \ldots,\left|\psi_{N}\right\rangle\left|e_{3}\right\rangle, \\
& \quad \vdots \\
& \left|\psi_{1}\right\rangle\left|e_{d_{m+1}}\right\rangle, \ldots,\left|\psi_{k}\right\rangle\left|e_{d_{m+1}}\right\rangle,\left|\psi_{k+1}\right\rangle\left|e_{d_{m+1}}\right\rangle, \ldots,\left|\psi_{N}\right\rangle\left|e_{d_{m+1}}\right\rangle
\end{aligned}
$$

are pairwise orthogonal to each other.

It is clearly that adding more states into a set increases the difficulty to discriminate them. Therefore, any set $\widetilde{\mathcal{S}}$ with $\mathcal{S} \subseteq \widetilde{\mathcal{S}} \subseteq \mathcal{S}_{\text {ext }}$ are also locally indistinguishable by LOCC. Notice that $|\widetilde{\mathcal{S}}|$ could be any integer lie between $k$ and $N d_{m+1}$. If $\mathcal{S}$ is completable, then $N$ can be chosen to be $\prod_{i=1}^{m} d_{i}$. Hence, $|\widetilde{\mathcal{S}}|$ could be any integer lie between $k$ and $\prod_{i=1}^{m+1} d_{i}$.

From the above argument, it is not difficult to show that if the original set $\left\{\left|\psi_{1}\right\rangle, \ldots,\left|\psi_{k}\right\rangle\right\}$ is completable, then $\left\{\left|\psi_{1}\right\rangle\left|\alpha_{1}\right\rangle, \ldots,\left|\psi_{k}\right\rangle\left|\alpha_{k}\right\rangle\right\}$ is also completable. For this setting (completable case), this approach of provides a large set of separable measurements, which cannot be accomplished by LOCC measurement.

Theorem 2. Let $\mathcal{S}_{o}=\left\{\left|\psi_{i}\right\rangle \mid 1 \leq i \leq k\right\}$ be a set of mutually orthogonal product states in $\mathbb{C}^{d_{1}} \otimes \mathbb{C}^{d_{2}} \otimes \cdots \otimes \mathbb{C}^{d_{m}}$. Suppose the set $\mathcal{S}_{o}$ is LOCC indistinguishable because all the local systems cannot start with a nontrivial measurement in order to preserve the orthogonality of these given states. Let $\left|\alpha^{(j)}\right\rangle,\left|\beta^{(j)}\right\rangle$ be two nonorthogonal states in $\mathbb{C}^{d_{j}^{\prime}}$ for $j=1,2, \ldots, n$. Then the set $\mathcal{S}$ of states

$$
\left\{\left|\psi_{1}\right\rangle \otimes\left(\otimes_{j=1}^{n}\left|\alpha_{1}^{(j)}\right\rangle\right), \ldots,\left|\psi_{k}\right\rangle \otimes\left(\otimes_{j=1}^{n}\left|\alpha_{k}^{(j)}\right\rangle\right)\right\}
$$

in $\left(\otimes_{i=1}^{m} \mathbb{C}^{d_{i}}\right) \otimes\left(\otimes_{j=1}^{n} \mathbb{C}^{d_{j}^{\prime}}\right)$ is also LOCC indistinguishable as $m+n$ parties where $\left|\alpha_{1}^{(j)}\right\rangle, \cdots,\left|\alpha_{k}^{(j)}\right\rangle \in\left\{\left|\alpha^{(j)}\right\rangle,\left|\beta^{(j)}\right\rangle\right\}$ for $j=1,2, \ldots, n$.

Proof: The idea of the proof is similar with that of theorem 1 . We call a set $S_{0}$ of mutually orthogonal product states in $\left(\otimes_{i=1}^{m} \mathbb{C}^{d_{i}}\right) \otimes\left(\otimes_{j=1}^{n} \mathbb{C}^{d_{j}^{\prime}}\right)$ shares the property $\mathcal{P}(m, n)$ if it satisfies the following two conditions:

(i) To preserve the orthogonality of $S_{0}$, each of the first $m$ parties could only do a trivial measurement.

(ii) For any state $S_{0}$, its local states of the last $n$ parties are just chosen from two pure nonorthogonal states of the corresponding system.

By assumption, the set $\mathcal{S}$ shares the property $\mathcal{P}(m, n)$. Thus, each of the first $m$ parties cannot be the first one to take a nontrivial measurement. Hence, one of the last $n$ systems should be the first to take the nontrivial measurement. However, by lemma 1, for any such measurement, there is an outcome such that the postmeasurement states $\mathcal{S}^{\prime}$ also share property $\mathcal{P}(m, n)$. To perfectly distinguish the states $\mathcal{S}$, we have to perfectly distinguish those states $\mathcal{S}^{\prime}$.
With the same argument, we could show that in order to perfectly distinguish the states $\mathcal{S}^{\prime}$, we have to perfectly distinguish another set $\mathcal{S}^{\prime \prime}$, which also shares property $\mathcal{P}(m, n)$. The significance is that this process cannot stop in any finite rounds of measurements. However, we are only allowed to play a finite rounds of measurements by the definition of LOCC. Hence, we deduce that the given set $\mathcal{S}$ is LOCC indistinguishable. Fig. 2 is a more intuitive figure to show the protocol presented in the proof.



Fig. 2: This is a sketch map to show the distinguishing protocol of theorem 2 with $n=3$. Here we use $A_{1}$, $A_{2}, \ldots, A_{m}$ to denote the first $m$ parties and $B_{1}, B_{2}, B_{3}$ to denote the last three parties. The significant point is that there exists an infinite sequence of outcomes (indicated by the red dotted line) performed by the last three parties. And these parties send a set with the $\mathcal{P}(m, 3)$ property to another set with the same property. Moreover, the post-measurement states along these outcomes are pictured by the grey rectangle.

Corollary 1. Suppose $\left\{\left|\psi_{1}\right\rangle, \ldots,\left|\psi_{k}\right\rangle\right\} \subseteq \mathbb{C}^{d_{1}} \otimes \mathbb{C}^{d_{2}} \otimes \cdots \otimes$ $\mathbb{C}^{d_{m}}$ is LOCC indistinguishable because no local system could play a nontrivial measurement in order to preserve the orthogonality of the given states. Let $\left|\alpha^{(j)}\right\rangle$ be any states in $\mathbb{C}^{d_{j}^{\prime}}$ for $j=1,2, \ldots, n$, then the set of states $\left\{\left|\psi_{1}\right\rangle \otimes_{j=1}^{n}\left|\alpha^{(j)}\right\rangle, \ldots,\left|\psi_{k}\right\rangle \otimes_{j=1}^{n}\left|\alpha^{(j)}\right\rangle\right\}$ is also LOCC indistinguishable as $m+n$ parties.

To better compare the result in corollary 1 with those in [31], here we quote one of the main results from reference [31]. "Suppose $\left\{|\varphi\rangle_{i}=|x\rangle_{i}|y\rangle_{i}, i=1,2, \ldots, l\right\}$ is a set of orthogonal product states in $d \otimes d$, which cannot be distinguished by LOCC because two parties cannot start a nontrivial measurement. Then, we give the following multipartite orthogonal product states in $d_{1} \otimes d_{2} \otimes \cdots \otimes d_{n}$ 


$$
\begin{gathered}
\left(d_{1,2, \ldots, n}=d \geq 3, n \geq 4\right) . \\
\left|\varphi_{i}\right\rangle=|x\rangle_{i}|y\rangle_{i}|b\rangle_{3} \cdots|b\rangle_{n-1}|a\rangle_{n}, \\
\left|\varphi_{i+l}\right\rangle=|x\rangle_{i}|a\rangle_{2}|y\rangle_{i} \cdots|b\rangle_{n-1}|b\rangle_{n}, \\
\vdots \\
\left|\varphi_{i+m l}\right\rangle=|x\rangle_{i}|b\rangle_{2} \cdots|a\rangle_{m+1}|y\rangle_{i} \cdots|b\rangle_{n}, \\
\vdots \\
\left|\varphi_{i+(n-2) l}\right\rangle=|x\rangle_{i}|b\rangle_{2} \cdots|b\rangle_{n-2}|a\rangle_{n-1}|y\rangle_{i}
\end{gathered}
$$

where $i=1,2, \cdots, l, 2 \leq m \leq n-3,|a\rangle_{j}$ is orthogonal to $|b\rangle_{j},(2 \leq j \leq n)$ in $d$-dimension quantum system." Then the theorem 1 of Ref. [31] asserts that the above $(n-1) l$ states cannot be perfectly distinguished by LOCC.

Fixed $m \in\{0, \ldots, n-2\}$, we can obtain that the set $\mathcal{S}_{m}$

$$
\left\{\left|\varphi_{i+m l}\right\rangle \mid i=1,2, \ldots, l\right\}
$$

cannot be distinguished by LOCC from corollary 1 . Therefore, the result of the corollary improves the results of study by Zhang [31] from the following two aspects.

(a) All the unit states $\left|\alpha^{(j)}\right\rangle \in \mathbb{C}^{d_{j}^{\prime}}$ are chosen arbitrarily and independently of each other.

(b) The set corresponding to the above corollary is only a subset of those in [31].

In [31], Zhang et al. showed that to preserve the orthogonality of states in $\bigcup_{m=0}^{n-2} \mathcal{S}_{m}$ any partite could only do a trivial measurement. Then they can obtain their statement. However, to preserve the orthogonality of states in $\mathcal{S}_{m}$ there are still some parties which could start with nontrivial measurement. Hence their methods fail to deal with the local distinguishability of $\mathcal{S}_{m}$.

It is natural to ask whether the results above can be generalized to cases with three or more nonorthogonal states. A "simply generalized" version of theorem 1 like replacing "Let $|\alpha\rangle,|\beta\rangle$ be two $\cdots \cdots\left|\alpha_{1}\right\rangle, \cdots,\left|\alpha_{k}\right\rangle \in$ $\{|\alpha\rangle,|\beta\rangle\}$ " in theorem 1 by "Let $|\alpha\rangle,|\beta\rangle,|\gamma\rangle$ be three $\cdots \cdots\left|\alpha_{1}\right\rangle, \cdots,\left|\alpha_{k}\right\rangle \in\{|\alpha\rangle,|\beta\rangle,|\gamma\rangle\} "$ may not be true, an example of which is provided below.

Example 2. With the same notation in equation (1), and let $|\alpha\rangle=|0\rangle,|\beta\rangle=\frac{1}{2}|0\rangle+\frac{\sqrt{3}}{2}|1\rangle,|\gamma\rangle=\frac{1}{2}|0\rangle-\frac{\sqrt{3}}{2}|1\rangle$. Then the set of states

$$
\left|\psi_{1}\right\rangle|\alpha\rangle,\left|\psi_{2}\right\rangle|\alpha\rangle,\left|\psi_{3}\right\rangle|\beta\rangle,\left|\psi_{4}\right\rangle|\beta\rangle,\left|\psi_{5}\right\rangle|\gamma\rangle,\left|\psi_{6}\right\rangle|\gamma\rangle
$$

is LOCC distinguishable.

Proof: Firstly, the fourth partite performs the measurement $\left\{M_{1}, M_{2}\right\}$ with

$$
M_{1}^{\dagger} M_{1}=\left[\begin{array}{cc}
\frac{1}{2} & \frac{1}{2 \sqrt{3}} \\
\frac{1}{2 \sqrt{3}} & \frac{1}{2}
\end{array}\right], M_{2}^{\dagger} M_{2}=\left[\begin{array}{cc}
\frac{1}{2} & \frac{-1}{2 \sqrt{3}} \\
\frac{-1}{2 \sqrt{3}} & \frac{1}{2}
\end{array}\right] .
$$

Clearly, $M_{1}^{\dagger} M_{1}+M_{2}^{\dagger} M_{2}=I_{2}$ and both $M_{1}^{\dagger} M_{1}$ and $M_{2}^{\dagger} M_{2}$ are semipositive definite matrices. Moreover, it can be easily verified that

$$
\left\langle\alpha\left|M_{1}^{\dagger} M_{1}\right| \gamma\right\rangle=0, \quad\left\langle\alpha\left|M_{2}^{\dagger} M_{2}\right| \beta\right\rangle=0 .
$$

According to these two equations, we have the following protocol to distinguish the six states in the example.

- If the measurement outcome is "1", Danny (the fourth system) continuously performs her measurement according to the basis $\left\{M_{1}|\alpha\rangle, M_{1}|\gamma\rangle\right\}$ (must be normalized). Moreover, the outcome " $\gamma$ " could eliminate the states $\left|\psi_{1}\right\rangle|\alpha\rangle,\left|\psi_{2}\right\rangle|\alpha\rangle$, and the first three parties could distinguish the other four. The outcome " $\alpha$ " could eliminate the states $\left|\psi_{5}\right\rangle|\gamma\rangle,\left|\psi_{6}\right\rangle|\gamma\rangle$; then, the first three parties could distinguish the remaining four states.

- If the measurement outcome is " 2 ", Danny continuously measures according to the basis $\left\{M_{2}|\alpha\rangle, M_{2}|\beta\rangle\right\}$. The outcome " $\beta$ " could eliminate the states $\left|\psi_{1}\right\rangle|\alpha\rangle,\left|\psi_{2}\right\rangle|\alpha\rangle$, and the first three parties could distinguish the other four. The outcome " $\alpha$ " could eliminate the states $\left|\psi_{5}\right\rangle|\beta\rangle,\left|\psi_{6}\right\rangle|\beta\rangle$, then the first three parties could distinguish the remaining four states.

Remark: It is much of interest to ask whether it is possible to generate new LOCC indistinguishable set by appending the system with three or more "appropriately selected" nonorthogonal states. Up to now, we haven't been able to solve this problem.

\section{CONCLUSION AND DISCUSSION}

In this paper, we present a new method for deriving sets of locally indistinguishable product states in multipartite quantum systems. The method is based on the following observation: given two nonorthogonal states; there exists at least one outcome of any measurement such that the post-measurement states corresponding to the two states are still nonorthogonal. Given any set of LOCC indistinguishable product states because all the local systems can not start with a nontrivial measurement, we can extend it by another partite with the local state being chosen from a set with two nonorthogonal states. However, with an additional partite joined in, the set is still LOCC indistinguishable. Based on this scheme, we show that it is possible to construct LOCC indistinguishable set with vary number of states. After that we generalize this with more parties joined in and show the target set is still LOCC indistinguishable. It can be seen that some parties can perform some nontrivial measurements. Hence, the sets are completely different from those constructed before. This result widen our knowledge of nonlocality without entanglement to some extent.

Moreover, we show that if the original given set is completable, the set with more parties is also completable. The local indistinguishability implies that these sets could be identified perfectly by separable operators. 
All these give nonlocal separable operations, that is, separable operations that cannot be implemented by LOCC.

There are also some interesting questions remain unsolved. For example, is it possible to generate new LOCC indistinguishable set by appending the system with three or more "appropriately selected" nonorthogonal states? If so, this would increase our freedom to construct set of locally indistinguishable product states.

Acknowledgments The authors thank the referee for asking several interesting questions to improve our previous version of this paper. The work is supported by the NSFC 11571119.

\section{APPENDIX A}

Proof of lemma 1: Since $\langle\alpha \mid \beta\rangle \neq 0$, we can write $|\beta\rangle$ as the form

$$
|\beta\rangle=\lambda|\alpha\rangle+\delta\left|\alpha^{\perp}\right\rangle, \quad \lambda \neq 0
$$

where $\left|\alpha^{\perp}\right\rangle \in \mathbb{C}^{d}$ is a unit vector and $\left\langle\alpha \mid \alpha^{\perp}\right\rangle=0$. Suppose $\left\{\left|\alpha_{1}\right\rangle,\left|\alpha_{2}\right\rangle, \cdots,\left|\alpha_{d}\right\rangle\right\}$ are orthonormal basis of $\mathbb{C}^{d}$ and $\left|\alpha_{1}\right\rangle=|\alpha\rangle,\left|\alpha_{2}\right\rangle=\left|\alpha^{\perp}\right\rangle$. Under this given basis, the matrix $M_{i}^{\dagger} M_{i}$ can be written as the form

$$
\left[\begin{array}{cccc}
m_{11}^{(i)} & m_{12}^{(i)} & \cdots & m_{1 d}^{(i)} \\
m_{21}^{(i)} & m_{22}^{(i)} & \cdots & m_{2 d}^{(i)} \\
\vdots & \vdots & \ddots & \vdots \\
m_{d 1}^{(i)} & m_{d 2}^{(i)} & \cdots & m_{d d}^{(i)}
\end{array}\right] .
$$

Suppose the claim of our lemma is not true. That is, for each $i$, at least one of the following three elements is zero:

$$
\left\langle\alpha\left|M_{i}^{\dagger} M_{i}\right| \alpha\right\rangle,\left\langle\alpha\left|M_{i}^{\dagger} M_{i}\right| \beta\right\rangle,\left\langle\beta\left|M_{i}^{\dagger} M_{i}\right| \beta\right\rangle .
$$

Hence the matrices $\left\{M_{i}^{\dagger} M_{i}\right\}_{i=1}^{n}$ can be separated into three classes as follows:

(i) $\left\langle\alpha\left|M_{i}^{\dagger} M_{i}\right| \alpha\right\rangle=0$ implies that $m_{11}^{(i)}=m_{12}^{(i)}=0$.

(ii) $\left\langle\alpha\left|M_{i}^{\dagger} M_{i}\right| \beta\right\rangle=0$ implies that $\lambda m_{11}^{(i)}+\delta m_{12}^{(i)}=0$.

(iii) $\left\langle\beta\left|M_{i}^{\dagger} M_{i}\right| \beta\right\rangle=0$ implies that $M_{i}|\beta\rangle=\mathbf{0}$. Hence $M_{i}^{\dagger} M_{i}|\beta\rangle=\mathbf{0}$. This also implies the relation between $m_{11}^{(i)}$ and $m_{12}^{(i)}: \lambda m_{11}^{(i)}+\delta m_{12}^{(i)}=0$.

Hence, we conclude that the three cases could give the same relation: $\lambda m_{11}^{(i)}+\delta m_{12}^{(i)}=0$. Since this holds for all $i \in\{1,2, \ldots, n\}$, we have

$$
\lambda \sum_{i=1}^{n} m_{11}^{(i)}+\delta \sum_{i=1}^{n} m_{12}^{(i)}=0 .
$$

On the other hand, the measurement definition naturally gives

$$
\sum_{i=1}^{n} M_{i}^{\dagger} M_{i}=I_{d}
$$

Particularly, by considering the entrywise equalities, we obtain

$$
\sum_{i=1}^{n} m_{11}^{(i)}=1, \sum_{i=1}^{n} m_{12}^{(i)}=0 .
$$

Then, equations (3) and (4) contradict each other as $\lambda \neq$ 0 . Hence, this completes the proof claimed in the lemma.
[1] M. A. Nielsen and I. L. Chuang, Quantum Computation and Quantum Information(Cambridge University Press, Cambridge, U.K., 2004).

[2] D.P. DiVincenzo, D.W. Leung and B.M. Terhal:, Quantum data hiding. IEEE Trans. Inf. Theory 48, 580 (2002).

[3] D. Markham and B. C. Sanders, Graph States for Quantum Secret Sharing. Phys. Rev. A 78, 042309 (2008).

[4] J. Walgate and L. Hardy, Nonlocality Asymmetry and Distinguishing Bipartite States, Phys. Rev. Lett. 89, 147901 (2002).

[5] J. Walgate, A. J. Short, L. Hardy, and V. Vedral, Local Distinguishability of Multipartite Orthogonal Quantum States, Phys. Rev. Lett. 85, 4972 (2000).

[6] S. Ghosh, G. Kar, A. Roy, A. Sen(De), and U. Sen, Distinguishability of Bell States, Phys. Rev. Lett. 87, 277902 (2001).

[7] H. Fan, Distinguishability and Indistinguishability by Local Operations and Classical Communication,
Phys. Rev. Lett. 92, 177905 (2004).

[8] M. Nathanson, Distinguishing bipartitite orthogonal states using LOCC: Best and worst cases, J. Math. Phys. (N.Y.) 46, 062103 (2005).

[9] H. Fan, Distinguishing bipartite states by local operations and classical communication, Phys. Rev. A 75,014305 (2007).

[10] S. M. Cohen, Local distinguishability with preservation of entanglement, Phys. Rev. A 75, 052313 (2007).

[11] S. Bandyopadhyay, S. Ghosh, and G. Kar, LOCC distinguishability of unilaterally transformable quantum states, New J. Phys. 13123013 (2011).

[12] N. Yu, R. Duan, and M. Ying, Four Locally Indistinguishable Ququad-Ququad Orthogonal Maximally Entangled States, Phys. Rev. Lett. 109, 020506 (2012).

[13] A. Cosentino, Positive partial transpose indistinguishable states via semidefinite programming, Phys. Rev. A 87, 012321 (2013). 
[14] M.-S. Li, Y.-L. Wang, S.-M. Fei and Z.-J. Zheng, $d$ locally indistinguishable maximally entangled states in $\mathbb{C}^{d} \otimes \mathbb{C}^{d}$, Phys. Rev. A 91, 042318 (2015).

[15] S. X. Yu and C. H. Oh, Detecting the local indistinguishability of maximally entangled states, arXiv:1502.01274v1.

[16] C. H. Bennett, D. P. DiVincenzo, C. A. Fuchs, T. Mor, E. Rains, P. W. Shor, J. A. Smolin, and W. K. Wootters, Quantum nonlocality without entanglement, Phys. Rev. A 59, 1070(1999).

[17] S. De Rinaldis, Distinguishability of complete and unextendible product bases, Phys. Rev. A 70, 022309 (2004).

[18] M. Horodecki, A. Sen(De), U. Sen, and K. Horodecki, Local Indistinguishability: More Nonlocality with Less Entanglement, Phys. Rev. Lett. 90, 047902 (2003).

[19] C. H. Bennett, D. P. DiVincenzo, T. Mor, P. W. Shor, J. A. Smolin, B. M. Terhal, Unextendible Product Bases and Bound Entanglement, Phys. Rev. Lett. 82, 5385 (1999).

[20] D. P. DiVincenzo, T. Mor, P. W. Shor, J. A. Smolin, and B. M. Terhal, Unextendible product bases, uncompletable product bases and bound entanglement, Comm. Math. Phys. 238, 379 (2003).

[21] Z.-C. Zhang, F. Gao, G.-J. Tian, T.-Q. Cao and Q.-Y. Wen, Nonlocality of orthogonal product basis quantum states, Phys. Rev. A 90, 022313 (2014).

[22] Z.-C. Zhang, F. Gao, S.-J. Qin, Y.-H. Yang, and Q.-Y. Wen, Nonlocality of orthogonal product states, Phys. Rev. A 92, 012332 (2015).

[23] Z.-C. Zhang, F. Gao, Y. Cao, S.-J. Qin, and Q.-Y. Wen, Local indistinguishability of orthogonal product states, Phys. Rev. A 93, 012314 (2016).

[24] G.-B. Xu, Y.-H. Yang, Q.-Y. Wen, S.-J. Qin, and F. Gao, Locally indistinguishable orthogonal product bases in arbitrary bipartite quantum system, Sci. Rep. 6, 31048 (2016).

[25] G.-B. Xu, Q.-Y. Wen, S.-J. Qin, Y.-H. Yang, and F. Gao, Quantum nonlocality of multipartite orthogonal product states, Phys. Rev. A 93, 032341 (2016).

[26] X.-Q. Zhang, X.-Q. Tan, J. Weng, and Y.-J. Li, LOCC indistinguishable orthogonal product quantum states, Sci. Rep. 6, 28864 (2016).

[27] Y.-L. Wang, M.-S. Li, Z.-J. Zheng, and S.-M. Fei, Nonlocality of orthogonal product-basis quantum states, Phys. Rev. A 92, 032313 (2015).

[28] Y.-L. Wang, M.-S. Li, Z.-J. Zheng, and S.-M. Fei, The local indistinguishability of multipartite product states, Quant. Info. Proc. 16, 5 (2017).

[29] Y. Feng and Y.-Y. Shi, Characterizing Locally Indistinguishable Orthogonal Product States, IEEE Trans. Inf. Theory 55, 2799 (2009).

[30] Y.-H. Yang, F. Gao, G.-J. Tian, T.-Q. Cao, and Q.-Y. Wen, Local distinguishability of orthogonal quantum states in a $2 \otimes 2 \otimes 2$ system, Phys. Rev. A 88, 024301 (2013).

[31] Z.-C. Zhang, K.-J. Zhang, F. Gao, Q.-Y. Wen, and C. H. Oh, Construction of nonlocal multipartite quantum states, Phys. Rev. A 95, 052344 (2017).

[32] X. Zhang, J. Weng, X. Tan, and W. Luo, Indistinguishability of pure orthogonal product states by LOCC, Quantum Inf. Process. 16, 168(2017).

[33] S. Halder, Several nonlocal sets of multipartite pure orthogonal product states, Phys. Rev. A 98, 022303 (2018).

[34] S. M. Cohen, Understanding entanglement as re- source: Locally distinguishing unextendible product bases, Phys. Rev. A 77, 012304 (2008).

[35] S.Bandyopadhyay, S. Halder, and M. Nathanson, Entanglement as a resource for local state discrimination in multipartite systems, Phys. Rev. A 94, 022311 (2016).

[36] Z.-C. Zhang, F. Gao, T.-Q. Cao, S.-J. Qin, and Q.-Y. Wen, Entanglement as a resource to distinguish orthogonal product states, Sci. Rep. 6, 30493 (2016).

[37] K. Feng, Unextendible product bases and 1-factorization of complete graphs, Disc. App. Math. 154, 942 (2006).

[38] N. Johnston, The structure of qubit unextendible product bases. J. Phys. A: Math. Theor. 47, 424034 (2014).

[39] Jianxin Chen and N. Johnston, The Minimum Size of Unextendible Product Bases in the Bipartite Case (and Some Multipartite Cases). Commun. Math. Phys. 333, 351-365 (2015). 